\title{
Real-World Outcomes Among Patients with Cystic Fibrosis Treated with Ivacaftor: 2012-2016 Experience
}

\author{
Mark Higgins · Nataliya Volkova $\cdot$ Kristin Moy • Bruce C. Marshall • \\ Diana Bilton
}

Received: February 28, 2020 / Published online: April 18, 2020

(C) The Author(s) 2020

\section{ABSTRACT}

Introduction: In this long-term, postapproval, observational study, data from the US Cystic Fibrosis Foundation Patient Registry and the UK Cystic Fibrosis Registry were used to evaluate the impact of ivacaftor treatment on cystic fibrosis (CF) by comparing outcomes in ivacaftor-treated patients with those in matched untreated comparator patients. Registry data

Enhanced Digital Features To view digital features for this article go to https://doi.org/10.6084/m9.figshare. 12011370 .

\author{
M. Higgins ( $\square)$ \\ Vertex Pharmaceuticals (Europe) Limited, 2 \\ Kingdom St, London W2 6BD, UK \\ e-mail: mark_higgins@vrtx.com \\ N. Volkova · K. Moy \\ Vertex Pharmaceuticals Incorporated, 50 Northern \\ Ave, Boston, MA, USA \\ B. C. Marshall \\ US Cystic Fibrosis Foundation, 4550 Montgomery \\ Ave, Suite 1100 N, Bethesda, MD, USA \\ D. Bilton \\ The Cystic Fibrosis Trust, 1 Aldgate, London EC3N \\ 1RE, UK \\ D. Bilton \\ National Heart and Lung Institute, Imperial College \\ London, London, UK \\ D. Bilton \\ Royal Brompton Hospital, London, UK
}

from up to 5 years of ivacaftor availability in the US and up to 4 years of availability in the UK were evaluated.

Methods: Starting in the first year of ivacaftor availability, ivacaftor-treated patients in each registry were matched $1: 5$ to comparator patients who never received ivacaftor. Clinical endpoints were evaluated in annual cross-sectional safety analyses. The key endpoints were death, organ transplants, pulmonary exacerbation, and hospitalization. Relative risks and 95\% CIs were calculated to compare the ivacaftor and comparator cohorts in each registry.

Results: Here, we report the complete and final results of the annual cross-sectional safety analyses across the duration of the study, with up to 5 years of follow-up. Data show a pattern of lower risk of death, transplant, pulmonary exacerbation, and hospitalization among ivacaftor-treated patients in both registries.

Conclusions: Ivacaftor-treated patients had consistently favorable clinical outcomes relative to untreated comparators, and no new safety concerns were identified. While general limitations of observational research apply, these findings support disease modification by $\mathrm{CF}$ transmembrane conductance regulator (CFTR) modulator therapy with ivacaftor. Future research of novel CFTR modulators will need to explore alternative methods for comparator selection for evaluation of clinical data given the evolving landscape of CF treatment. 


\section{PLAIN LANGUAGE SUMMARY}

We performed a study to better understand the long-term impact of treatment with a drug called ivacaftor for patients with cystic fibrosis (CF). Our study used data from CF patient registries in the United Kingdom and the United States. These registries collect information about patients with $\mathrm{CF}$, their health, and the treatments they receive. Using data from these registries, we compared patients treated with ivacaftor with a similar group of patients (similar age, sex, and disease severity) who did not receive ivacaftor. We looked at the clinical outcomes of each group every year for up to 5 years. In the final analysis from our study, we found no new safety concerns associated with ivacaftor treatment. Additionally, we found that patients treated with ivacaftor tended to have lower risks of death, organ transplant, pulmonary exacerbations, and hospitalizations. Overall, these results demonstrate the favorable impact of ivacaftor treatment on long-term outcomes of patients with CF.

Keywords: Cystic fibrosis; Ivacaftor; Real-world data; Registry; Long-term safety

\section{Key Summary Points}

Why carry out this study?

In cystic fibrosis (CF), a life-shortening, rare genetic disease, researchers have the unique opportunity to evaluate data from the majority of patients in their respective regions collected by national CF registries, with the US Cystic Fibrosis Foundation Patient Registry (US CFFPR) and the UK Cystic Fibrosis Registry (UK CFR) being the largest.
To evaluate disease progression and clinical outcomes in patients treated with ivacaftor (the first approved CF transmembrane conductance regulator [CFTR] modulator targeting the underlying cause of $\mathrm{CF}$ ), we conducted a long-term observational study comparing outcomes in patients in the US and UK registries who were treated with ivacaftor versus matched untreated comparator patients.

This study asked, "Among patients with CF treated with ivacaftor in real-world clinical practice, what are the patterns in key clinical outcomes?"

\section{What was learned from the study?}

Analyses of up to 5 years of experience with ivacaftor in real-world clinical practice across countries and years identified no new safety concerns and demonstrated consistently favorable outcomes in ivacaftor-treated patients.

These data support the conclusion that highly effective CFTR modulation with ivacaftor leads to disease modification, consistent with the multisystem benefits observed in clinical trials.

\section{INTRODUCTION}

Real-world data provide information about long-term safety and effectiveness of treatments outside of clinical trials; the value of such data is increasingly recognized as real-world analysis methodologies become more rigorous [1]. Disease registries are a valuable source of real-world data and are useful to evaluate benefits and risks of medicines [2]. In cystic fibrosis (CF), a lifeshortening, rare genetic disease, researchers have the unique opportunity to evaluate data from the majority of patients in their respective regions collected by national CF registries, with the US Cystic Fibrosis Foundation Patient Registry (US CFFPR) and the UK Cystic Fibrosis 
Registry (UK CFR) being the largest [3-6]. These registries provide a window into long-term outcomes in patients in the real-world setting $[7,8]$.

To evaluate disease progression and clinical outcomes in patients treated with the first approved CF transmembrane conductance regulator (CFTR) modulator targeting the underlying cause of $\mathrm{CF}$, we conducted a long-term observational study comparing outcomes in patients in the US and UK registries who were treated with ivacaftor versus matched untreated comparator patients $[7,8]$. This postauthorization safety surveillance study was designed to fulfill a postmarketing commitment to the European Medicines Agency and is disclosed on the European Union electronic Register of PostAuthorization Studies (EUPAS4270) [7, 8]. Following previous reports, including the previously published disease progression analyses $[7,8]$, we report final results of the annual cross-sectional safety analyses across the duration of the study. These data provide insights to address the aim of assessing patients' long-term outcomes with highly effective CFTR modulation with ivacaftor in real-world clinical practice.

\section{METHODS}

\section{Study Population and Analysis Cohorts}

The study populations have been previously described [7, 8]. Starting in the first year of ivacaftor availability (2012 in the United States; 2013 in the United Kingdom), ivacaftor-treated patients in the US CFFPR and UK CFR were

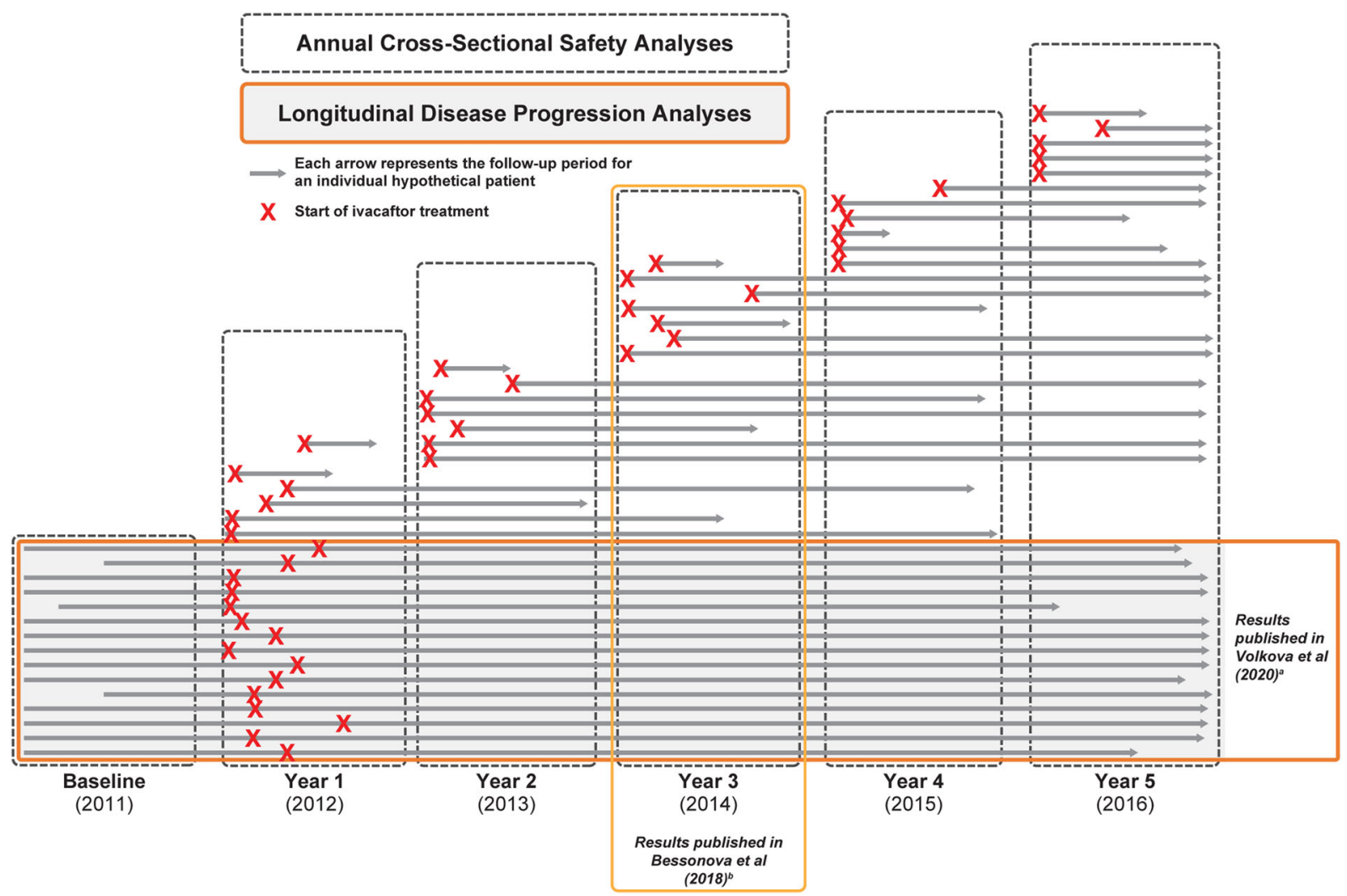

Fig. 1 Study design. US ivacaftor patient cohorts analyzed in the annual cross-sectional safety analyses and longitudinal disease progression analyses are shown. Comparator patients were matched to ivacaftor-treated patients $(5: 1)$ for age, sex, and CFTR genotype severity. A similar approach was used for UK patients. ${ }^{\text {a}}$ Volkova et al. [8]. ${ }^{\mathrm{b}}$ Bessonova et al. [7] 
matched $1: 5$ by age, sex, and CFTR mutation genotype severity (predominantly classes I-III) to comparator patients who never received ivacaftor. Outcomes in ivacaftor versus comparator cohorts were analyzed separately in each registry, both in annual cross-sectional safety analyses (focusing on patient outcomes during a single year; Fig. 1) and in longitudinal analyses of disease progression outcomes over time $[7,8]$. The annual cross-sectional safety analyses were conducted based on patients included in the annual safety cohorts: the ivacaftor safety cohort in the first year of the study included all patients treated with ivacaftor in the first year of availability; the ivacaftor safety cohort in subsequent years' analyses included patients who were members of the ivacaftor safety cohort the preceding year, were still alive, continued to receive ivacaftor, and remained in the registry at the beginning of the year, as well as any patients with a new record of ivacaftor treatment during that analysis year. Similarly, the annual comparator safety cohorts included patients who were members of the same cohort the previous year, were still alive, had no record of ivacaftor or lumacaftor/ivacaftor use, and remained in the registry at the beginning of the year, as well as new patients (with no record of ivacaftor or lumacaftor/ivacaftor use) who were matched to the new patients in that year's ivacaftor safety cohort. In contrast, the longitudinal disease progression analyses focused on the disease progression cohorts, i.e., the subset of patients followed continuously from the first year of availability through study completion. Details and complete results from the longitudinal disease progression analyses have been reported elsewhere [8].

\section{Data Analysis}

Clinical endpoints evaluated in the annual cross-sectional safety analyses included death, organ transplants, pulmonary exacerbations, and hospitalizations; registry definitions rather than clinical trial definitions were used. Death was defined as evidence of a date of death in the respective registry database. Organ transplants were defined as evidence of organ transplant in the registry database, including heart/lung, lung alone, liver, kidney, and other transplants. Pulmonary exacerbations were defined as episodes requiring intravenous antibiotic use at home or in the hospital. Hospitalizations were defined differently across the two registries: in the US CFFPR, hospitalization data included patients hospitalized for any reason (pulmonary exacerbation, pulmonary complication, gastrointestinal complication, transplant-related, sinus infection, nontransplant surgery, or other reasons), whereas in the UK CFR, only hospitalizations for pulmonary exacerbation were collected.

Relative risks and 95\% CIs, based on normal approximation, were calculated to compare the ivacaftor and comparator cohorts in each registry. If the expected frequency was $<5$ in $\geq 1$ substratum of the contingency table, Fisher's exact $P$ values were calculated in lieu of CIs. Cross-sectional analyses were performed separately for each registry due to differences in data capture.

\section{Ethics Compliance}

As this is an analysis of an existing de-identified dataset, it is exempt from institutional review board (IRB) approval. Data from the US CFFPR and the UK CFR were used in this study with permission of each registry. Patient information was obtained and maintained by the CF Trust (in the United Kingdom) and CF Foundation (in the United States) patient registries, which obtain informed consent as part of their registry enrollment procedures. In the United Kingdom, the CF Trust Patient Registry consent procedures have been agreed upon with the National Research Ethics Service. The registry is registered under the Data Protection Act (1998) and has Research Ethics Committee approval. In the United States, data for the CF Foundation registry are only collected at sites where IRB approval has been obtained and for subjects for whom consent or assent (as applicable) is obtained. 
Table 1 Annual safety cohort sample sizes

\begin{tabular}{|c|c|c|c|c|c|}
\hline & 2012 & 2013 & 2014 & 2015 & 2016 \\
\hline \multicolumn{6}{|l|}{ US CFFPR } \\
\hline $\begin{array}{l}\text { Ivacaftor safety } \\
\text { cohort, } n\end{array}$ & 807 & 999 & 1256 & 1727 & 1858 \\
\hline $\begin{array}{l}\text { Comparator safety } \\
\text { cohort, } n^{\text {a }}\end{array}$ & 4035 & 4932 & 6200 & 7329 & 7316 \\
\hline \multicolumn{6}{|l|}{ UK CFR } \\
\hline $\begin{array}{l}\text { Ivacaftor safety } \\
\text { cohort, } n\end{array}$ & NA & 307 & 411 & 432 & 462 \\
\hline $\begin{array}{l}\text { Comparator safety } \\
\text { cohort, } n\end{array}$ & NA & 1533 & 2069 & 2201 & 2372 \\
\hline \multicolumn{6}{|c|}{$\begin{array}{l}N A \text { not applicable } \\
\text { a Increased rates of attrition were observed in the US } \\
\text { comparator cohort in } 2015 \text { and } 2016 \text { and were primarily } \\
\text { driven by the exclusion of patients with a new record of } \\
\text { lumacaftor/ivacaftor therapy after it became commercially } \\
\text { available in the United States in } 2015\end{array}$} \\
\hline
\end{tabular}

\section{RESULTS}

The number of patients receiving ivacaftor therapy in the US CFFPR increased from 807 during year 1 (2012) to 1858 during year 5 (2016). In the UK CFR, the number of patients receiving ivacaftor therapy increased from 307 during year 1 (2013) to 462 during year 4 (2016). The number of patients in each safety cohort in each analysis year are shown in Table 1.

Results of all annual cross-sectional safety analyses were generally consistent across time and countries. Relative risks of death, organ transplant, pulmonary exacerbation, and hospitalization in the ivacaftor safety cohort versus the comparator safety cohort of each registry in each analysis year are shown in Table 2 along with the corresponding 95\% CIs.

No new safety concerns with ivacaftor were identified. Favorable clinical outcomes across multiple endpoints were observed in patients

Table 2 Summary of key safety outcomes across US and UK annual analyses of ivacaftor versus comparator safety cohorts

\begin{tabular}{|c|c|c|c|c|c|}
\hline $\begin{array}{l}\text { Relative risk }(95 \% \mathrm{CI}) / \\
\text { Fisher's exact } \boldsymbol{P} \text { value }\end{array}$ & 2012 & 2013 & 2014 & 2015 & 2016 \\
\hline \multicolumn{6}{|l|}{ US CFFPR } \\
\hline Death & $0.43(0.17,1.07)$ & $0.37(0.15,0.93)$ & $0.41(0.20,0.84)$ & $0.18(0.07,0.44)$ & $0.41(0.20,0.86)$ \\
\hline Organ transplants & $0.22(0.05,0.89)$ & $0.19(0.05,0.76)$ & $0.15(0.04,0.59)$ & $0.55(0.27,1.09)$ & $0.29(0.13,0.67)$ \\
\hline Pulmonary exacerbation & $0.70(0.62,0.78)$ & $0.62(0.56,0.69)$ & $0.64(0.58,0.70)$ & $0.69(0.63,0.76)$ & $0.72(0.66,0.79)$ \\
\hline Hospitalizations $^{\mathrm{b}}$ & $0.67(0.60,0.76)$ & $0.59(0.53,0.66)$ & $0.64(0.58,0.70)$ & $0.66(0.60,0.72)$ & $0.67(0.62,0.73)$ \\
\hline \multicolumn{6}{|l|}{ UK CFR } \\
\hline Death & NA & $0.45, P=0.41$ & $0.52(0.16,1.70)$ & $0.75(0.27,2.15)$ & $0.47, P=0.41$ \\
\hline Organ transplants & NA & $0.76, P=1.00$ & $0.56, P=0.56$ & NA, $P=0.06$ & $0.24, P=0.20$ \\
\hline Pulmonary exacerbation & NA & $0.89(0.79,1.00)$ & $0.61(0.53,0.70)$ & $0.61(0.53,0.70)$ & $0.58(0.50,0.67)$ \\
\hline $\begin{array}{l}\text { Hospitalization due } \\
\text { to pulmonary exacerbation }\end{array}$ & NA & $0.87(0.75,1.01)$ & $0.57(0.48,0.68)$ & $0.62(0.53,0.73)$ & $0.57(0.48,0.67)$ \\
\hline
\end{tabular}

$N A$ not applicable

a Fisher's exact $P$ values are shown when the expected value is $<5$ in $\geq 1$ cell of the contingency table

b Reasons for hospitalization included pulmonary exacerbation, pulmonary complication, gastrointestinal complication, transplant related, sinus infection, nontransplant surgery, and other 
treated with ivacaftor relative to untreated comparators; data show a pattern of lower risk of death, transplant, pulmonary exacerbation, and hospitalization among ivacaftor-treated patients in both registries. This is consistent with the previously reported preservation of lung function in ivacaftor-treated patients versus comparators [8]. Overall, these results demonstrate the favorable impact of ivacaftor on several markers of CF disease.

\section{DISCUSSION}

Analyses of up to 5 years of experience with ivacaftor in real-world clinical practice across countries and years identified no new safety concerns and demonstrated consistently favorable outcomes in ivacaftor-treated patients. These results are consistent with and build upon previous reports from this study [7, 8]. The first report from this study described results from the 2014 annual cross-sectional safety analysis (i.e., year 3 in the United States and year 2 in the United Kingdom) with similar results to those reported here [7]; however, whereas that report described a single snapshot of data, we have now demonstrated that the observed favorable trends for ivacaftor treatment were durable and consistent across 4 to 5 years of analysis. Together, results from the annual cross-sectional safety analyses reported here and results from the long-term longitudinal disease progression analysis [8] reinforce the favorable long-term outcomes of ivacaftor treatment, both in terms of patients' annual experience during each year of treatment and when considering their cumulative experience over time. These data support the conclusion that highly effective CFTR modulation with ivacaftor leads to disease modification, consistent with the multisystem benefits observed in clinical trials [9-12].

A lower risk of death was observed relatively early on and remained consistent each year. A similar finding was observed for the risk of organ transplant. Reductions in the annual risk of other clinically relevant outcomes, including hospitalizations and pulmonary exacerbations, were also observed. It should be emphasized that the beneficial patterns for all outcomes evaluated in this study were consistently observed across the analysis years in each registry, even though the annual relative risk estimates varied from year to year, which was not unexpected given the changing composition of cohorts included in each annual cross-sectional analysis inherent to the study design.

It should also be noted that all the outcome patterns were consistent between the two registries, even though there were differences in definitions used by the registries for some outcomes. For instance, although hospitalizations were defined differently in the two registries (hospitalizations due to any reason were considered in the US CFFPR, whereas only hospitalizations due to pulmonary exacerbations were considered in the UK CFR), the patterns observed were similar between the two registries, and the difference in definitions is not expected to impact the conclusions of the study since most hospitalizations in this population are driven by pulmonary exacerbations $[4,13]$

This study is not without limitations inherent to observational research in general. These include lack of standardized assessment and visit schedules as well as a lack of precise dates for treatment and event occurrences. Additionally, because uptake of ivacaftor in eligible patients was so high, we used a concurrent comparator cohort with different genotypes but comparable disease severity $[8,14,15]$. Although patients were matched for age, sex, and CFTR genotype severity, the difference in genotypes between cohorts may have impacted patients' prognoses in unknown ways. Patients' baseline characteristics did not suggest any such difference in baseline prognoses, and subsequent analyses of outcomes were stratified by age, sex, and percent predicted forced expiratory volume in $1 \mathrm{~s}$ to mitigate potential confounding factors $[7,8]$; however, the possibility of confounding factors must be acknowledged. There were also high attrition rates in the last 2 years of follow-up in the US CFFPR comparator cohort due to the availability of lumacaftor/ ivacaftor therapy for patients homozygous for the F508del mutation (resulting in their exclusion from this study). However, it is important to note that this attrition most likely resulted in an underestimation of the ivacaftor treatment 
benefit due to the fact that the excluded patients tended to be those who were older and had more severe disease [8].

Real-world research can be complementary to clinical trials, illustrating outcomes among patients receiving ivacaftor in real-world practice. This study relied on data from two registries, which used distinct data-capture methodologies. Despite this, we were able to make use of the data set from each registry to analyze comparable outcomes and obtain confirmation that the trends we observed were consistent. In addition, trends observed from these real-world outcomes are extremely consistent with outcomes from several prior studies evaluating ivacaftor treatment $[7,8]$.

Selection of comparator groups in $\mathrm{CF}$ research can be challenging because there are many CF-causing CFTR mutations, and the burden of illness varies between genotypes [14]. In future research of novel CFTR modulators indicated for broader patient populations, identification of concurrent comparators may not be feasible, and alternative approaches, such as historical comparators, may need to be explored. In addition, continuity of and adherence to therapy and the possibility of patients switching between different CFTR modulators will be important considerations.

\section{CONCLUSIONS}

Overall, patients treated with ivacaftor had consistently favorable clinical outcomes relative to their untreated comparators, and no new safety concerns were identified. These data demonstrate the long-term impact of highly effective CFTR modulation with ivacaftor.

\section{ACKNOWLEDGEMENTS}

The authors thank the Cystic Fibrosis Foundation and the Cystic Fibrosis Trust for the use of patient registry data to conduct this study. Additionally, the authors thank the patients, care providers, and clinic coordinators at $\mathrm{CF}$ centers throughout the United States and the
United Kingdom for their contributions to the patient registries.

Funding. This work was supported by Vertex Pharmaceuticals Incorporated. Vertex Pharmaceuticals Incorporated developed the protocol (with approval from the European Medicines Agency) and statistical analysis plan and prepared the study report based on analyses performed by the registry partners. Vertex Pharmaceuticals Incorporated also funded the journal's Rapid Service Fee.

Authorship. All named authors meet the International Committee of Medical Journal Editors (ICMJE) criteria for authorship for this article, take responsibility for the integrity of the work as a whole, and have given their approval for this version to be published.

Medical writing, editorial, and other assistance. Tejendra Patel, PharmD, of Vertex Pharmaceuticals Incorporated, provided editorial support and coordination. Medical writing and editorial support were provided by Karen Kaluza Smith, PhD, of ArticulateScience LLC, which received funding from Vertex Pharmaceuticals Incorporated.

Disclosures. All authors received nonfinancial support (assistance with manuscript preparation) from ArticulateScience LLC, which received funding from Vertex Pharmaceuticals. Additional disclosures are as follows: Mark Higgins and Nataliya Volkova: employees of Vertex Pharmaceuticals and may own stock or stock options in Vertex Pharmaceuticals. Kristin Moy: former employee of Vertex Pharmaceuticals and may own stock or stock options in Vertex Pharmaceuticals; employee of Alexion Pharmaceuticals. Bruce C Marshall: the CFF has entered into therapeutic development award agreements and licensing agreements to assist with the development of CFTR modulators that may result in intellectual property rights, royalties, and other fees provided to CFF by various pharmaceutical companies. Diana Bilton: member of the Steering Committee of the UK CFR, which provided data for this study. 
Compliance with Ethics Guidelines. As this is an analysis of an existing de-identified dataset, it is exempt from Institutional Review Board (IRB) approval. Data from the US Cystic Fibrosis Foundation Patient Registry and the UK Cystic Fibrosis Registry were used in this study with permission of each registry. Patient information was obtained and maintained by the CF Trust (in the United Kingdom) and CF Foundation (in the United States) patient registries, which obtain informed consent as part of their registry enrollment procedures. In the United Kingdom, the CF Trust Patient Registry consent procedures have been agreed upon with the National Research Ethics Service. The registry is registered under the Data Protection Act (1998) and has Research Ethics Committee approval. In the United States, data for the CF Foundation registry are only collected at sites where IRB approval has been obtained and for subjects for whom consent or assent (as applicable) is obtained.

Prior presentation. The data in this manuscript were previously presented in poster format at the European Cystic Fibrosis Conference in 2017 and 2018 (Bessonova L, et al. Presented at the 40th European Cystic Fibrosis Conference; June 7-10, 2017; Seville, Spain. Poster EPS5.10; and Volkova N, et al. Presented at the 41st European Cystic Fibrosis Conference; June 6-9, 2018; Belgrade, Serbia. Poster IPD2.02).

Data availability. Data sharing is not applicable to this article as no datasets were generated or analyzed during the current study. Each registry collects and manages its own data and maintains processes for researchers to request data (US CFFPR: https://www.cff.org/ Research/Researcher-Resources/Tools-and-Reso urces/Patient-Registry-Data-Requests/; UK CFR: https://www.cysticfibrosis.org.uk/the-work-wedo/uk-cf-registry/apply-for-data-from-the-uk-cfregistry).

Open Access. This article is distributed under the terms of the Creative Commons Attribution-NonCommercial 4.0 International License (http://creativecommons.org/licenses/ by-nc/4.0/), which permits any non- commercial use, distribution, and reproduction in any medium, provided you give appropriate credit to the original author(s) and the source, provide a link to the Creative Commons license, and indicate if changes were made.

\section{REFERENCES}

1. Blonde L, Khunti K, Harris SB, Meizinger C, Skolnik NS. Interpretation and impact of real-world clinical data for the practicing clinician. Adv Ther. 2018;35: 1763-74.

2. McGettigan P, Alonso Olmo C, Plueschke K, et al. Patient registries: an underused resource for medicines evaluation : operational proposals for increasing the use of patient registries in regulatory assessments. Drug Saf. 2019;42:1343-51.

3. Knapp EA, Fink AK, Goss $\mathrm{CH}$, et al. The Cystic Fibrosis Foundation Patient Registry. Design and methods of a national observational disease registry. Ann Am Thorac Soc 2016;13:1173-9.

4. Cystic Fibrosis Foundation Patient Registry. 2018 annual data report. Bethesda: Cystic Fibrosis Foundation; 2019.

5. Taylor-Robinson D, Archangelidi O, Carr SB, et al. Data Resource Profile: The UK Cystic Fibrosis Registry. Int J Epidemiol. 2018;47:9-10e.

6. Cystic Fibrosis Trust. UK Cystic Fibrosis Registry: annual data report 2016. London: Cystic Fibrosis Trust; 2017.

7. Bessonova L, Volkova N, Higgins M, et al. Data from the US and UK cystic fibrosis registries support disease modification by CFTR modulation with ivacaftor. Thorax. 2018;73:731-40.

8. Volkova N, Moy K, Evans J, et al. Disease progression in patients with cystic fibrosis treated with ivacaftor: data from national US and UK registries. J Cyst Fibros. 2020;19:68-79.

9. Ramsey BW, Davies J, McElvaney NG, et al. A CFTR potentiator in patients with cystic fibrosis and the G551D mutation. N Engl J Med. 2011;365: 1663-722.

10. Davies JC, Wainwright CE, Canny GJ, et al. Efficacy and safety of ivacaftor in patients aged 6 to 11 years with cystic fibrosis with a G551D mutation. Am J Respir Crit Care Med. 2013;187:1219-25. 
11. Davies JC, Cunningham S, Harris WT, et al. Safety, pharmacokinetics, and pharmacodynamics of ivacaftor in patients aged 2-5 years with cystic fibrosis and a CFTR gating mutation (KIWI): an open-label, single-arm study. Lancet Respir Med. 2016;4: 107-15.

12. Rosenfeld M, Wainwright CE, Higgins $M$, et al. Ivacaftor treatment of cystic fibrosis in children aged 12 to less than 24 months and with a CFTR gating mutation (ARRIVAL): a phase 3 single-arm study. Lancet Respir Med. 2018;6:545-53.
13. Newton TJ. Respiratory care of the hospitalized patient with cystic fibrosis. Respir Care. 2009;54: 769-75.

14. McKone EF, Emerson SS, Edwards KL, Aitken ML. Effect of genotype on phenotype and mortality in cystic fibrosis: a retrospective cohort study. Lancet. 2003;361:1671-6.

15. Sawicki GS, McKone EF, Millar SJ, et al. Patients with cystic fibrosis and a G551D or homozygous F508del mutation: similar lung function decline. Am J Respir Crit Care Med. 2017;195:1673-6. 